\title{
Plastic deformations in thin rotational shells
}

\author{
Avgustina Astakhova ${ }^{1, *}$ \\ ${ }^{1}$ Moscow State University of Civil Engineering, Yaroslavskoye sh. 26, 129337 Moscow, Russia
}

\begin{abstract}
The paper focuses on the method of calculation of evolution shells beyond the elastic limit. The conclusion of the basic system of differential equations is based on the linear shell theory with regard to the Hirchhoff-Lave hypothesis and on the physical equations for small elasticplastic deformation theory using the method of elastic decisions. The boundary conditions are formulated for Cauchy problem: rigid attachment, hinged support, and free margin. The spherical shell boundary conditions in the pole are obtained from the conditions of symmetry and antisymmetry functions. The convergence of the elastic method and the method of the occurrence of superficial plastic deformations are studied. Also the stress-strain state in the spherical shell is determined and the convergence of the obtained solutions was studied. The results are presented on the symmetric load ring applied to the middle of the Meridian and on the load that can be considered as a concentrated force. The sufficient quantity of iterations is established to achieve the accuracy of $0.1 \%$. The graphs are presented for radial displacement and for meridional bending moment as the functions that converge more rapidly and more slowly respectively.
\end{abstract}

\section{State of the problem}

The work is dedicated to the calculation of the isotopic shells of revolution of constant thickness under the action of symmetrical and assimetrical loads beyond the elastic limit. Long and short, closed and ring shells are considered with different hypothesis about contour intersection. The conclusion of the basic system of differential equations is based on the linear shell theory with regard to the Hirchhoff-Lave hypothesis and on the physical equations for small elastic-plastic deformation theory using the method of elastic decisions [1-3].

It is supposed that the shell mid-surface is the surface of rotation about the axis $z$ without special points escluding points where the speciality is prevented. Shell generator is a quite smooth curve that curvature is too small comparing to $1 / h$.

Figure 1 indicates:

$\theta$ - azimuthal angle in the horizontal plane,

$S$ - meridian arc length,

$\varsigma$ - normal to the mid-surface.

\footnotetext{
* Corresponding author: ast956@yandex.ru
} 
On the basis of the linear theory and Kirchhoff-Lave hypothesis the deformations are presented as two first power series expentions at $\varsigma$, i.e. excluding values above the second order of smallness.

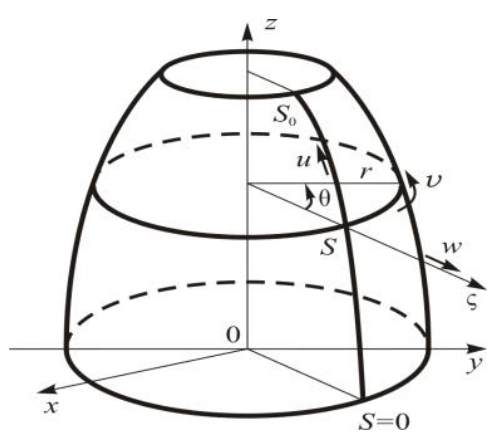

Fig. 1. Shell mid-surface.

\section{The method of solution}

The stress-strain state of thin shells is determined with the use of the elastic solutions method based on small elastic-plastic deformations. I. S. Tsurkov developed elastic solutions method in relation to the analysis of shells that was proposed by A. A. Ilyushin [4]. On the basis of the theory, all the generalized efforts are presented in the form of the difference of efforts that occurs in the linearly deformed structure $\mathbf{N}^{0}$ and incremental efforts $\Delta$ that takes into account the physical nonlinearity of the material:

$$
\mathbf{N}=\mathbf{N}^{0}-\Delta
$$

The resolving system of differential equations is derived from equilibrium equations, geometric relations of linear theory, and physical equations for small elastic-plastic deformations theory:

$$
\begin{aligned}
& \sigma_{i}=E \varepsilon_{i}, \quad \varepsilon_{i}<\varepsilon_{s}^{0}, \\
& \sigma_{i}=E \varepsilon_{i}\left[1-\lambda\left(1-\frac{\varepsilon_{s}^{0}}{\varepsilon_{i}}\right)\right], \quad \varepsilon_{i}>\varepsilon_{s}^{0}, \quad \lambda=\frac{E-E_{1}}{E} .
\end{aligned}
$$

All the components of the stress-strain state and displacements decompose to Fourier series. Eight resolution functions are introduced to the system. The original system of partial differential equations is transformed to the system of eight ordinary differential equations for the first order with respect to the Fourier series coefficients of resolution functions [2]:

$$
\mathbf{N}^{\mathbf{0}}=\mathbf{A}(s) \times \mathbf{N}+\mathbf{f}(s)+\mathbf{B}(s) \times \mathbf{\Delta}
$$

Here: $\mathbf{A}(s)=a_{i j}, \mathbf{B}(s)=b_{\mathrm{ik}}-$ constant coefficients $(i, j=1,2, \ldots, 8 ; l, k=1,2, \ldots, 6), \quad \mathbf{N}$ $=\left(N_{r}, N_{z}, \hat{S}, M_{s}, u_{r}, u_{z}, v, \zeta_{s}\right), \mathbf{f}(s)=\left(f_{1}, f_{2}, f_{3}, f_{4}, 0,0,0,0\right), f_{1}=-q_{r}, f_{2}=-q_{z}, f_{3}=-q_{\theta}$, $f_{4}=-m_{s}, \quad \Delta=\left(\Delta N_{s}, \Delta N_{\theta}, \Delta M_{s}, \Delta M_{\theta}, \Delta S, \Delta H\right)$. 
Resolution functions are: $N_{r}$ - radial force, $N_{z}$ - force along the axis $z$ (axial force), $\hat{S}-$ generalized tangential force, $M_{s}$ - meridional bending moment, $u_{r}$ - radial displacement, $u_{z}$ - axial displacement, $v$ - tangential displacement, $\zeta_{s}-$ angular deflection. Furthermore, shell deformation is defined by the formulas:

$$
\mathbf{E}=\mathbf{C}(s) \cdot \mathbf{N}+\mathbf{D}(s)
$$

Here: $\mathbf{C}(s)=c_{i j}, \mathbf{D}(s)=d_{l k}-$ constant coefficients, $\mathbf{E}=\left(\varepsilon_{s}, \varepsilon_{\theta}, \varepsilon_{s \theta}, \kappa_{s}^{0}, \kappa_{\theta}^{0}, \kappa^{0}{ }_{s \theta}\right)$.

Elastic solutions method is based on the successive approximations method. In the first approximation incremental efforts $\Delta$, that take into account physical nonlinearity of the material, are considered to be equal to zero, i.e. the elastic problem is resolved. The system of ordinary differential equations (3) should be integrated by the orthogonal sweep method to obtain $n$ Fourier series expansions. Thus, eight resolution functions are determined by the coefficients, and the functions are used for determining six deformation components (1.4): relative stretch deformations $\left(\varepsilon_{s}, \varepsilon_{\theta}\right)$, deformations of shift $\left(\varepsilon_{s \theta}\right)$, flexural deformations $\left(\kappa_{s}^{0}\right.$, $\left.\kappa_{\theta}^{0}\right)$ and torsional deformations $\left(\kappa_{s \theta}^{0}\right)$.

Four boundary conditions at each end of the generating line should be specified for the solution of Cauchy problem:

1) rigid attachment: $u_{r}=u_{z}=v=0, \quad \zeta_{s}=0$;

2) hinged support: $u_{r}=u_{z}=v=0, M_{s}=0$;

3) free margin: $N_{r}=N_{z}=\hat{S}=M_{s}=0$.

In the pole of the spherical shell boundary conditions can be obtained from the conditions of symmetry and antisymmetry functions in a neighborhood of the point $r=0$ and from the equations that can be obtained with using passage to the limit $r \rightarrow 0$ in a geometric ratio and equilibrium equations [5]:

$$
\begin{array}{ll}
u_{r}=v=0, \quad N_{z}=0, \quad \zeta_{s}=0 & \text { for } n=0, \\
u_{r}+v=0, \quad u_{z}=0, \quad M_{s}=0 & \text { for } n=1, \\
u_{r}=u_{z}=v=0, \quad \zeta_{s}=0 & \text { at the even } n, \\
M_{s}=0 & \text { at the odd } n \text { for } n>2
\end{array}
$$

Dimensionless loads and displacements are used in all the equations:

$$
\left(P, N_{r}, N_{z}, \hat{S}\right) / E h, \quad\left(M_{s}, M_{\theta}\right) / E h^{2}, \quad\left(u_{r}, u_{z}, v\right) / h \text {. }
$$

\section{Results}

Moreover, the intensities of the deformations on the external $\varepsilon_{i 2}$ and on the internal $\varepsilon_{i 1}$ surfaces of the shell are related to the $\varepsilon_{s}{ }^{0}$ relative deformation that corresponds to the yield strength $\sigma_{\mathrm{T}}:\left(\varepsilon_{i 1}, \varepsilon_{i 2}\right) / \varepsilon_{s}^{0}$.

In the result of the regional and local effects located in the thin shells of revolution, the desired efforts have large gradients. That's why to solve the problem for numerical implementation the discrete orthogonalization method was used. Direct integration was conducted by Runge-Kutta method with a stable step of integration along the shell generatrix. Load corrections $\Delta$ were only at the nodes of orthogonalization. The calculation results show that the difference in the function values is $0.5 \%$ at 50 orthogonalization points and 10 integration points at each orthogonalization step. The results coinside with an accuracy of $0.1 \%$ at 50 and 25 points of orthogonalization and at 250 integration points.

In the present work the stress-strain state in the spherical shell was determined and the convergence of the obtained solutions was studied. Also the majority of the tasks were handled with 250 integration points and with 50 orthogonalization points. As a criterion for convergence of the solution, we used the criterion where every tenth approximation is compared for all resolution functions at all $\mathrm{N}$-points of orthogonalization. In the result a 
positive convergence of the method was discovered. It should be noted that the convergence of the solutions is too slow for the load range. The more extended the area of plastic deformations on the shell surface, the more approximations are required to achieve the same accuracy of solution.

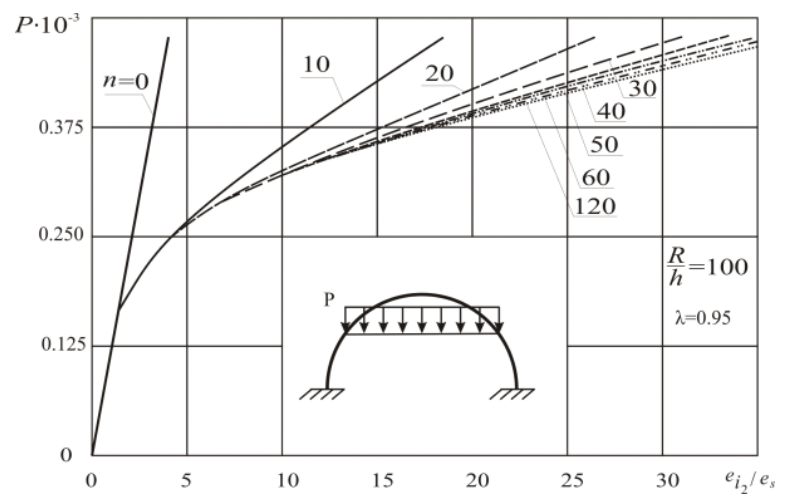

Fig. 2. Rotational shell under the action of the rotation load.

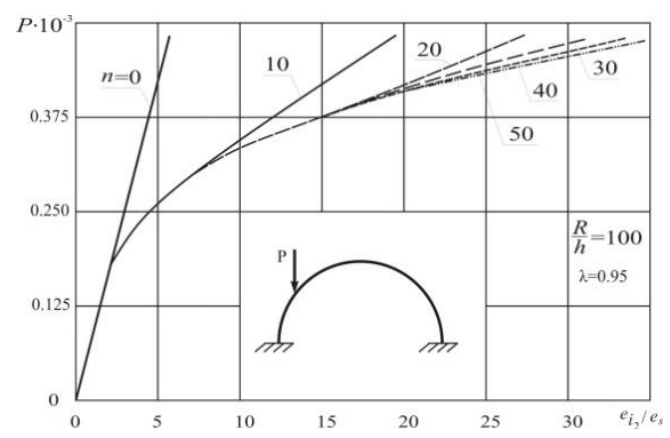

Fig. 3. Rotational shell under the action of the concentrated force.

The results presented in Fig. 2 and 3 refer to the spherical shell with the semi opening angle $\alpha=90^{\circ}$ and radius ratio to the thickness $R / h=100$. The steel chart tensile 3 approximated by the broken line with the hardening module $\lambda=0.95$ is presented. The intensity of deformations corresponding to the beginning of yielding is equal to $\varepsilon_{s}{ }^{0}=0.001$. Fig. 2 shows the dependence $\varepsilon_{i 2} / \varepsilon_{s}-P$ on the symmetric load ring applied to the middle of the Meridian. Thus, the elastic solution is suitable only if the load does not exceed $P / E h=$ $0.1510^{-3}$. To achieve the precision of $0.1 \% 10$ approximations with $P / E h<0.25 \cdot 10^{-3}$ and 100 approximations with $P / E h>0.35 \cdot 10^{-3}$ are required.

Fig. 3 shows the dependence $\varepsilon_{i 2} / \varepsilon_{s}-P$ on the load ring operating along the parallel arc, the corresponding angle $\theta=0.04 \mathrm{rad}$. The angle is small, so the load can be considered as a concentrated force. To achieve the accuracy of $0.1 \%$ at $P / E h<0.3 \cdot 10^{-3}$ it is enough to establish 10 approximations, and at $P / E h>0.45 \cdot 10^{-3}$ it is enough to establish 50 approximations. 
Fig. 5. Changes in the functions of the radial displacements and the meridional bending moments.

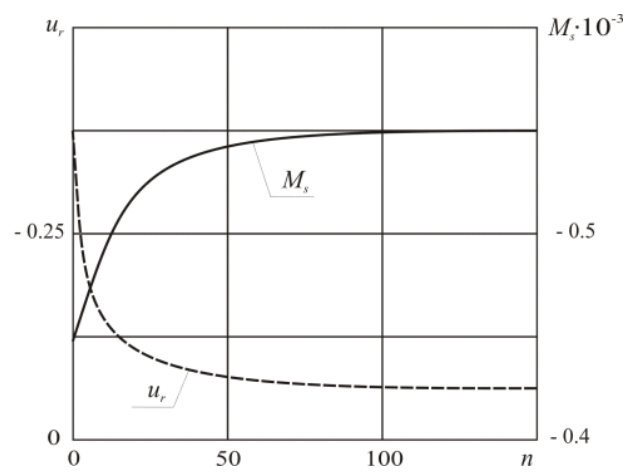

Fig. 4. Change of radial displacements functions and meridional bending moment.

Fig.4 shows the change of functions of the radial displacements $u_{r} / h$ and meridional bending moment $M_{s} / E h^{2}$ at the point of application of load depending on the number of approximation $n$ under the action of load $P / E h=0.2862 \cdot 10^{-3}$. The solid line is the change in the meridional bending moment, and the dashed line represents the change in the radial displacement. Based on these studies with the load $P / E h=0.2862 \cdot 10^{-3}$ it is possible to see two results. First, the radial displacement $u_{r}$ is the function that converges more rapidly where the accuracy $0.1 \%$ is achieved by the approximations with $n=10-20$ iterations in the solution. Second, the meridional bending moment $M_{s}$ is the function that converges slowly, that's why to achieve the accuracy $0.1 \% n=80-90$ iterations are required.

The studies show that the convergence of the elastic solutions method slows with the increase of the linear hardening coefficient $\lambda$, i.e. with the approaching to the Prandtl diagram. 40 iterations at $\lambda=0.95,100$ iterations at $\lambda=0.992$ and 150 iterations at $\lambda=1$ are required considering the example of the changes in the functions of the radial displacements $u_{r} / h$ and of the meridional bending moments $M_{s} / E h^{2}$ at the point-of-load application (Fig.5) for $P / E h=0.2862 \cdot 10^{-3}$.

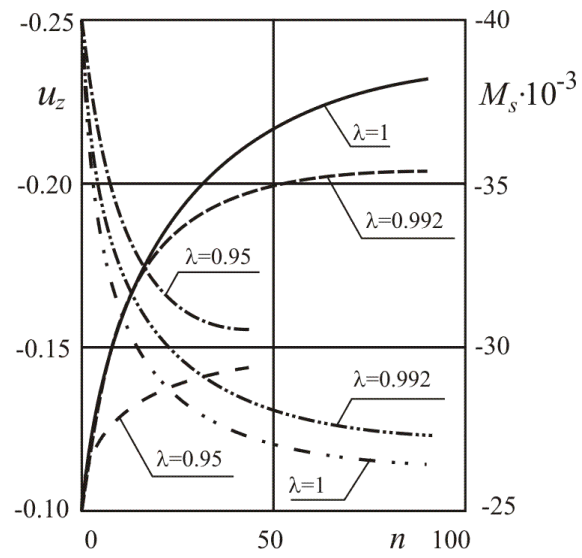

Fig. 5. Changes in the functions of the radial displacements and the meridional bending moments.

Figure 6 shows the areas of plastic deformation in the membranes of different thickness with radius to thickness ratio $R / h=200,100,50,25$ and with the ring load $P / E h=$ $0.2862 \cdot 10^{-3}$. The areas of plastic deformations are located near the point of force application. 


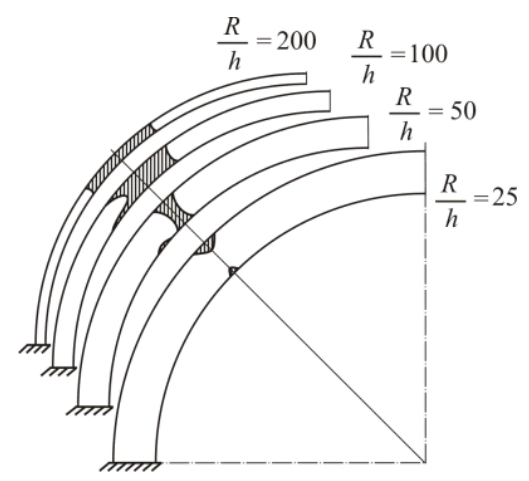

Fig. 6. Areas of plastic deformations in the membranes of different thickness.

Based on the calculation results, the dependences (Fig. 7) of the axial displacement $u_{z}$ were constructed by the load for the shells with radius to thickness ratio $R / h=200,100,50$, 25 . Here the elastic solution is direct, the other lines are calculated considering the plastic work of the material. The graphs allow to determine the load to the quantity of which the elastic solution is true.

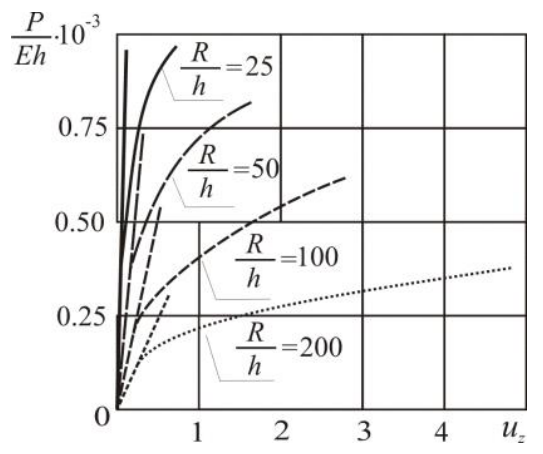

Fig. 7. Dependences of axial displacement.

\section{Conclusions}

The results are received on the base of the elastic solutions method. The method of the occurrence of superficial plastic deformations is studied. The results of the studying allow to define the load range at which elastic-plastic deformations appear. The areas of elasticplastic deformations extension for different thickness ratio $R / h=200,100,50,25$ on the load ring.

\section{References}

1. V.Z. Vlasov, Shell theory and its application to the technics (Gostekhizdat, Moscow, 1949)

2. Y.M. Grigorenko, F.T. Vasilenko, Applied mechanics 3, (1968)

3. A.A. Gorshkov, A.Y. Astakhova, N.Yu. Tsybin, Fundamentals of elastic thin shells(NIU MSUCE, Moscow, 2016)

4. I.S. Tsurkov, Collected papers of MISI named after V.V.Kuibyshev 47, (1965) 
5. A.Y. Astakhova, Calculation of elastoplastic shells under fixed load (MTT, Moscow, (1985) 